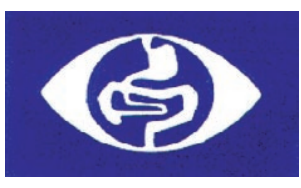

ESGE (European Society of Gastrointestinal Endoscopy)

\title{
European Society of Gastrointestinal Endoscopy Guideline for Video Capsule Endoscopy
}

\author{
J-F. Rey ${ }^{1}$ \\ G. Gay ${ }^{2}$ \\ A. Kruse ${ }^{3}$ \\ R. Lambert ${ }^{4}$ \\ ESGE Guidelines Committee
}

\section{Introduction}

Video capsule endoscopy (VCE) represents a significant advance in the investigation of intestinal diseases. The performance of the procedure and the indications for it are reviewed here in order to establish guidelines for its use, in accordance with current knowledge based on clinical practice and the published literature. In this guideline, all of the technical features described refer to the M2A Given Imaging video capsule (Given Imaging, Ltd., Yoqneam, Israel).

Video capsule endoscopy is a new technique in which a disposable video capsule is used that is swallowed by the patient. This allows visualization of the segment of the small bowel that is not within the reach of standard upper and lower endoscopy. The main current indication for capsule endoscopy is an obscure source of gastrointestinal bleeding. VCE may be particularly helpful in identifying the cause of bleeding when standard upper endoscopy and colonoscopy examinations are negative.

\section{Description of the Procedure}

The entire VCE procedure can be conducted on an outpatient basis. The patient is instructed to fast for $12 \mathrm{~h}$ before the procedure. The patient wears a shoulder-supported belt pack holding a power supply containing five nickel-metal $1.2-\mathrm{V}$ batteries (the size of D cells), and a small 305-gigabyte hard drive for storing the received images. Before ingestion of the capsule, a sensor array is taped to the patient's anterior abdominal wall in a designat- ed pattern and connected to the hard drive. Correlation between the findings in the gastrointestinal lumen and the general location of the capsule in the abdomen is attempted by triangulating the signal received from the three closest sensor ports.

Patients are allowed to drink clear liquids $1 \mathrm{~h}$ after ingestion of the capsule and to eat a light meal $4 \mathrm{~h}$ after ingestion. During the recording period, patients are instructed to record abdominal symptoms in a diary and are requested to check the blinking light on the belt pack periodically to confirm that the signal is being received. After $8 \mathrm{~h}$, the sensor array and the data recorder are removed, and the recorded digital information is downloaded to a computer workstation. The capsule is excreted naturally, usually within 24 to $48 \mathrm{~h}$.

Data are downloaded from the belt pack recorder to a customized PC workstation. This process takes $2-3 \mathrm{~h}$. Proprietary software is then used to review the images in an adjustable rapidscanning mode that can display between 1 and 25 frames per second. For subsequent access, thumbnail video clips of segments incorporating 50 images before and after an identified finding can be annotated and saved. Reading and interpreting the images requires an average of $40-60 \mathrm{~min}$.

There are a few issues that are still unresolved with regard to the procedure and patient preparation. The expert consensus recommendation is that the patient should always receive preparation with bowel cleansing using 2 liters of polyethylene glycol, administered either on the evening before the recording or on the same day at least $2-3 \mathrm{~h}$ before ingestion of the capsule. The

Institution

${ }^{1}$ Department of Hepatology and Gastroenterology, Institute Arnault Tzanck, St. Laurent du Var, France

${ }^{2}$ Internal Medicine Department I, CHU de Brabois, Vandoeuvre-les-Nancy, France

${ }^{3}$ Department of Surgical Gastroenterology, Aarhus University Hospital, Aarhus, Denmark

${ }^{4}$ Internal Agency for Research on Cancer, Lyon, France

Corresponding Author

J-F. Rey, M. D. · Department of Hepatology and Gastroenterology · Institute Arnault Tzanck · Avenue du Dr. Maurice Donat · 06700 St. Laurent du Var·France·Fax: +33-4-93075158 ·E-mail: jean-francois.rey@wandoo.fr

Bibliography

Endoscopy 2004; 36 (7): 656-658 @ Georg Thieme Verlag KG Stuttgart · New York · ISSN 0013-726X

DOI 10.1055/s-2004-814557 
advantage of preparation is that it cleanses the terminal ileum, allowing a better examination.

\section{Reading the Recorded Images}

\section{Localization}

The workstation software used to process the capsule images incorporates a localization feature. This makes it possible to estimate the location of any pathological findings within an average range of $3.77 \mathrm{~cm}$. An attempt is made to identify the capsule's location in the abdomen by triangulating the three closest sensors, selected according to the strength of the signals received from the capsule by the eight sensors attached to the abdominal wall. The location is then calculated from the position of these sensors and displayed on the computer screen as a two-dimensional image. However, in clinical practice, the software identification of the location of lesions is often considered to be too imprecise, and the location is mainly assessed on the basis of the distinct mucosal patterns visualized in the jejunum and ileum.

\section{Suspected Bleeding Indication}

The software application now includes an indicator feature (the "Suspected Blood Indicator"), which automatically marks images that correlate with suspected blood or red areas in the small intestine. This is helpful for identifying bleeding sites during the reading process. The clinical relevance of this software feature has yet to be evaluated.

\section{Multiview}

The software application has also recently had a "Multiview" feature added for reading of the VCE recordings. This allows simultaneous display of two images from the recording in separate, adjacent windows.

\section{Indications}

\section{Obscure Digestive Bleeding}

The most frequent indication for VCE is currently to diagnose obscure bleeding. Obscure bleeding is defined as either recurrent episodes of gastrointestinal bleeding - e.g., with melena or hematochezia - or a positive fecal occult blood test, or chronic iron-deficiency anemia, after negative findings on gastroscopy and colonoscopy.

Before VCE, patients with obscure gastrointestinal bleeding should undergo one more upper gastrointestinal endoscopy examination, conducted by an expert using a lateral-viewing endoscope, to exclude missed lesions within the reach of the endoscope (such as postpyloric or postbulbar ulcers), as well as lesions in the third and fourth parts of the duodenum. The reported diagnostic yield of VCE has ranged from $55 \%$ to $81 \%$. In comparative studies, VCE has been found to be consistently superior to other examination methods for diagnosing the source of gastrointestinal bleeding.

\section{Other Indications Currently Accepted}

Investigation of Crohn's disease

There is growing acceptance of the use of VCE in patients with Crohn's disease. Several comparative studies have evaluated the diagnostic yield of VCE and compared it with various radiographic modalities. VCE is able to detect intestinal lesions in a large subset of patients with known Crohn's disease, and it can also often detect intestinal lesions in patients in whom there is a clinical and/or biological suspicion of Crohn's. The diagnostic yield ranges from $43 \%$ to $71 \%$. VCE frequently extends the area of the bowel diagnosed as being affected by the disease, or detects lesions compatible with the diagnosis. However, these results need to be carefully analyzed, for several reasons: firstly, in most reports VCE has been compared with small-bowel follow-through examinations, which are not the most sensitive radiographic method; secondly, the patients included in the various studies may have different clinical situations in which the outcome of intestinal investigations needs to be assessed in different ways; thirdly, most studies have included limited numbers of patients; fourthly, it is not clear from these reports whether the diagnosis of Crohn's disease was confirmed afterwards with biopsies. Clinical trials are currently being conducted to evaluate these indications. On the other hand, it might be worth using the M2A Patency Capsule (Given Imaging, Ltd., Yoqneam, Israel) in patients with a higher risk of intestinal stenosis.

\section{Evaluation of the side effects of nonsteroidal anti-inflamma- tory drugs (NSAIDs)}

Several studies have shown that intestinal lesions are frequent in patients treated with NSAIDs. The clinical picture may include overt or occult bleeding, abdominal pain, or intestinal obstruction in cases of stenosis. The lesions most frequently found are ulcers and erosions.

\section{Hereditary polyposis syndromes}

The use of VCE may be considered for the surveillance of hereditary polyposis syndromes, such as familial adenomatous polyposis (FAP), in which a biopsy is needed in order to assess the risk, and familial juvenile polyposis.

\section{Prospective Indications Complicated celiac disease}

VCE may be of interest in the diagnostic work-up patients with celiac disease who present with atypical symptoms or chronic iron-deficiency anemia. In patients with a known diagnosis of celiac disease, VCE is indicated in cases of symptomatic relapse despite compliance with a gluten-free diet. In this situation, VCE may reveal a pattern of ulcerative jejunitis or T-cell lymphoma.

\section{Pediatric capsule endoscopy}

A few reports so far published have evaluated the feasibility and clinical usefulness of VCE in children. VCE is safe in children approximately 9 years of age or older. In younger patients, there have been a few tests involving endoscopic release of the capsule in the stomach. 


\section{Overall Tolerance and Contraindications}

The overall tolerance of VCE by patients has been impressively good in all studies. The capsule should be used with caution in patients with known or suspected gastrointestinal obstruction, fistulas, and swallowing disorders. Recent data suggest that the radiofrequency signal in VCE does not interfere with cardiac pacemakers. Safety in pregnancy has not yet been established.

\section{Obstruction of Capsule Progression}

A tight stenosis or large polyp may obstruct the progression of the capsule and require treatment. In all situations in which there is thought to be a risk, such as polyposis syndromes, NSAID complications, or Crohn's disease, it should be regarded as mandatory to have the patient complete a questionnaire regarding any intestinal symptoms that might represent contraindications. Studies are currently being conducted to assess the clinical value of an initial sham test with the M2A Patency System (which uses a dissolvable capsule) before the real capsule examination.
VCE is an exciting new diagnostic facility for assessing the lumen of the digestive tract. However, at present it is clearly only an adjunct to upper and lower gastrointestinal endoscopy. It is generally preferable to time-consuming diagnostic enteroscopy, and may lead to further developments in therapeutic enteroscopy. It is currently the preferred test for mucosal imaging of the entire small intestine. The absence of reimbursement for the procedure in major European countries is still a major drawback. 